Dept. of Math. Univ. of OsLo

Pure Mathematics

ISBN $82-553-1361-3 \quad$ No. 1

ISSN 0806-2439 January 2003

\title{
ON ARBITRAGE-FREE PRICING OF WEATHER DERIVATIVES BASED ON FRACTIONAL BROWNIAN MOTION
}

\author{
FRED ESPEN BENTH
}

\begin{abstract}
We derive arbitrage-free pricing dynamics for claims on temperature, where the temperature follows a fractional Ornstein-Uhlenbeck process. Using a fractional white noise calculus, we can express the dynamics as a special type of conditional expectation not coinciding with the classical one. Using a Fourier transformation technique, explicit expressions are derived for claims of European and average type, and we show that these pricing formulas are solutions of certain Black \& Scholes partial differential equations. Our results partly confirm a conjecture made by Brody, Syroka and Zervos [3].
\end{abstract}

\section{INTRODUCTION}

Weather derivatives have gained increasing popularity in financial markets as a tool to hedge weather risk. Many weather related products are traded in a buy-and-hold fashion, simply because there are no second-hand market for the contracts. However, markets for more structured products are emerging (e.g., the CME in London), and this calls for dynamical models of the derivatives in order to understand their risk exposure. Weather markets are typical examples of so-called incomplete markets since the underlying weather factor cannot be used for trading purposes.

The main objective of this paper is to present arbitrage-free models for derivatives on temperature. Temperature has a significant memory effect in its time evolution, together with a mean-reversion towards a seasonal variation. Empirical studies suggest an OrnsteinUhlenbeck process driven by a fractional Brownian motion to model temperature (see Brody, Syroka and Zervos [3]). Fractional Brownian motion is not a semimartingale, and the classical financial theory for incomplete markets breaks down. However, resorting to recent progress on a fractional stochastic calculus based on chaos expansion and a different interpretation of fractional stochastic integration (see Duncan, Hu and Pasik-Duncan [8] and $\mathrm{Hu}$ and Øksendal [9]), one can generalize the martingale-based financial theory in order to derive arbitrage-free dynamics. Our framework will provide price dynamics as a solution of a Black \& Scholes like partial differential equation or as a convolution product between the payoff function and a heat kernel.

Brody, Syroka and Zervos [3] derive prices for different temperature options as solutions of partial differential equations (see Kopp [12] for related work). They calculate the price at

Date: January 17, 2003.

Key words and phrases. Fractional Brownian motion, weather derivatives, arbitrage, option pricing, partial differential equations, white noise analysis. 
the time when the contract is entered, but not the dynamics of the option value. However, they conjecture that this price is given as a conditional expectation and solves a certain partial differential equation. We partly confirm their conjecture, although using a slightly different notion of conditional expectation. It turns out that we can derive arbitrage-free price dynamics using the so-called quasi-conditional expectation. The quasi-conditional expectation is an operator on the chaos expansion of square integrable random variables, and possesses many of the properties of the classical conditional expectation, however being different from it except for the case of Brownian motion. Although the definition of the quasi-conditional expectation seems to be quite abstract (as an operator on the chaos expansion), we can derive explicit expressions for it in many interesting cases together with associating it as solutions of partial differential equations. A peculiar result of our theory is that prices of derivatives no longer depends on the time to exercise, but on the current time and the exercise time. We discuss this further in the paper.

Necula [14] derives a dynamics for options on assets modeled as a fractional geometric Brownian motion (see e.g. Hu and Øksendal [9]). The author uses a Fourier approach to generalize the Black \& Scholes Formula and to show that European call/put options on the assets have a pricing dynamics being the solution of a fractional version of the Black \& Scholes partial differential equations. Our results generalize this to the weather market where the underlying dynamics are different. We prove that the prices of the derivatives are arbitrage-free. In addition, we clarify some results concerning the Fourier transform and quasi-conditional expectation and consider Asian options which are more relevant in markets trading weather products.

We remark that the evaluation problem of weather related derivatives can be attacked using a utility optimization approach instead. This view is taken in Davis [4], where he prices weather derivatives by relying on an approximation of the accumulated Heating degree days ${ }^{1}$ as a geometric Brownian motion.

The paper is organized as follows: In Section 2 we present some results from the white noise analysis for fractional Brownian motion, and derive some useful properties of the quasi-conditional expectation. The next Section deals with a fractional model for temperature dynamics, and we derive a dynamics for the average temperature as well. Moving on to Section 4, we prove that the quasi-conditional expectation provides the framework for deriving arbitrage-free prices for derivatives (or claims) on temperature. In Section 5 we calculate the prices for European and Asian claims, and in Section 6 we end the paper with identifying the prices as solutions of modulated Black \& Scholes partial differential equations.

\footnotetext{
${ }^{1}$ Heating degree days (HDD) is defined as $\max (18-X(t), 0)$, while Cooling degree days (CDD) is $\max (X(t)-18)$. Here, $X(t)$ is the temperature at time $t$. When temperature is lower than $18^{\circ} \mathrm{C}$, heating systems are turned on, while temperatures above $18^{\circ} \mathrm{C}$ require cooling from air-conditioners. The choice of $18^{\circ} \mathrm{C}$ is conventional.
} 


\section{A FRACTIONAL CALCULUS AND QUASI-MARTINGALES}

We recall some theory of fractional white noise calculus developed by Duncan, Hu and Pasik-Duncan [8] and $\mathrm{Hu}$ and Øksendal [9], together with some minor extensions concerning quasi-conditional expectations. Once the correct notion of fractional stochastic integration has been introduced, the fractional version of white noise analysis is parallel to the theory presented in Hida, Kuo, Potthoff and Streit [7]. Many of the extensions on quasi-conditional expectation that we do have their analogy in Benth and Potthoff [1].

Let $(\Omega, \mathcal{F}, P)$ be a complete probability space supporting a fractional Brownian motion $B_{H}(t)$ with Hurst parameter $\frac{1}{2}<H<1$, and denote by $\mathcal{F}_{t}^{H}$ the $\sigma$-algebra generated by $B_{H}(s)$ for $s \leq t$. Introduce the weight function

$$
\phi(s, t)=H(2 H-1)|s-t|^{2 H-2}, \quad s, t \in \mathbb{R},
$$

and let the Hilbert space $L_{\phi}^{2}\left(\mathbb{R}^{n}\right)$ be the set of functions $f: \mathbb{R}^{n} \mapsto \mathbb{R}$ such that

$$
|f|_{\phi, n}^{2}:=\langle f, f\rangle_{\phi, n}<\infty
$$

where the inner product $\langle\cdot, \cdot\rangle_{\phi, n}$ is

$$
\langle f, g\rangle_{\phi, n}=\int_{\mathbb{R}^{n} \times \mathbb{R}^{n}} f\left(s_{1}, \ldots, s_{n}\right) g\left(t_{1}, \ldots, t_{n}\right) \phi\left(s_{1}, t_{1}\right) \cdots \phi\left(s_{n}, t_{n}\right) d s d t .
$$

For a symmetric function $f \in L_{\phi}^{2}\left(\mathbb{R}^{n}\right)$, define the iterated fractional Wiener integral as

$$
I_{n}(f)=\int_{\mathbb{R}^{n}} f d B_{H}^{\otimes n}=n ! \int_{s_{1}<\cdots<s_{n}} f\left(s_{1}, \ldots, s_{n}\right) d B_{H}\left(s_{1}\right) \cdots d B_{H}\left(s_{n}\right) .
$$

We state the fractional chaos expansion theorem for random variables with finite variance:

Theorem 2.1. If $X \in L^{2}(\Omega, \mathcal{F}, P)$, then there exists a sequence of symmetric functions $f_{n} \in L_{\phi}^{2}\left(\mathbb{R}^{n}\right)$ such that

$$
X=\sum_{n=0}^{\infty} I_{n}\left(f_{n}\right)
$$

and

$$
E\left[X^{2}\right]=\sum_{n=0}^{\infty} n !\left|f_{n}\right|_{\phi, n}^{2}
$$

Frequently, we shall use the shorthand notation $L^{2}(P)$ when we mean $L^{2}(\Omega, \mathcal{F}, P)$.

Following the argument in Hida [6, Prop. 4.5], a stochastic process $X(t)=\sum_{n=0}^{\infty} I_{n}\left(f_{n}(t ; \cdot)\right)$ with finite variance is $\mathcal{F}_{t}^{H}$-adapted if and only if $\operatorname{supp} f_{n}(t ; \cdot) \subset[0, t]$. The definition of a stochastic integral with respect to fractional Brownian motion is motivated from the white noise theory (see e.g. Hida, Kuo, Potthoff and Streit [7]):

Definition 2.1. Let $X(s) \in L^{2}(P), 0 \leq s \leq t$, be an $\mathcal{F}_{s}^{H}$-adapted stochastic process with chaos expansion $X(s)=\sum_{n=0}^{\infty} I_{n}\left(f_{n}(s ; \cdot)\right)$. The fractional Itô integral of $X(t)$ is defined to 
be

$$
\int_{0}^{t} X(s) d B_{H}(s):=\sum_{n=0}^{\infty} I_{n+1}\left(\frac{1}{n+1} \tilde{f}_{n} 1_{[0, t)}^{\otimes(n+1)}\right)
$$

where the function $\tilde{f}_{n}$ is the symmetrization of $f_{n}(\cdot ; \cdot)$. The stochastic integral defines a random variable (or, more precisely, a stochastic process) in $L^{2}(P)$.

We remark that nowhere in the definition we used that the integrand is adapted. In fact, the above definition holds for anticipating integrands and is sometimes referred to as the Skorohod integral. We shall not dwell in any more detail on this generalized integral, but proceed with a Lemma connecting our definition of the fractional stochastic integral with the one given by Duncan, Hu and Pasik-Duncan [8] and Hu and Øksendal [9]:

Lemma 2.2. Let $X(s) \in L^{2}(P), 0 \leq s \leq t$, be an $\mathcal{F}_{s}^{H}$-adapted stochastic process with chaos expansion $X(s)=\sum_{n=0}^{\infty} I_{n}\left(f_{n}(s ; \cdot)\right)$. Assume $s \mapsto X(s) \diamond W_{H}(s)$ is Pettis integrable, where $\diamond$ is the Wick product and $W_{H}$ fractional white noise ${ }^{2}$. Then

$$
\int_{0}^{t} X(s) d B_{H}(s)=\int_{0}^{t} X(s) \diamond W_{H}(s) d s .
$$

The integral on the right-hand side is interpreted in the Pettis sense.

Proof. The proof uses machinery from the fractional white noise analysis described in detail by $\mathrm{Hu}$ and $\varnothing \mathrm{ksendal}[9]$. It holds that $W_{H}(s)=I_{1}\left(\delta_{s}\right), \delta_{s}$ being the Dirac $\delta$-function at time $s$. By definition of the Wick product and Pettis integration, we find

$$
\begin{aligned}
\int_{0}^{t} X(s) \diamond W_{H}(s) d s & =\int_{0}^{t} I_{n+1}\left(f_{n}\left(s ; \cdot \hat{\otimes} \delta_{s}(\cdot)\right) d s=I_{n+1}\left(\int_{0}^{t} f_{n}(s ; \cdot) \hat{\otimes} \delta_{s}(\cdot) d s\right)\right. \\
& =\frac{1}{n+1} I_{n+1}\left(\tilde{f}_{n} 1_{[0, t)}^{\otimes(n+1)}\right)
\end{aligned}
$$

This shows the Lemma.

This way of defining stochastic integration was first suggested by Duncan, Hu and PasikDuncan [8] (in a slightly different, but completely analgous manner), and paved the way for a fractional white noise calculus as presented in $\mathrm{Hu}$ and $\varnothing \mathrm{ksendal}[9]$ and in the present paper.

With the notion of stochastic integration at hand, we can state a version of the fractional Itô Formula found in Duncan, $\mathrm{Hu}$ and Pasik-Duncan [8]. We use the notation $f_{x}=$ $\partial f / \partial x, f_{x x}=\partial^{2} f / \partial x^{2}$ etc. for a differentiable function $f$ :

Theorem 2.3. Assume $X(t)=\int_{0}^{t} a(s) d B_{H}(s)$, where $a \in L_{\phi}^{2}(\mathbb{R})$. For a function $f \in$ $C^{1,2}\left(\mathbb{R}_{+} \times \mathbb{R}\right)$ with bounded derivatives we have

$$
f(t, X(t))=f(0,0)+\int_{0}^{t} f_{t}(s, X(s)) d s+\int_{0}^{t} f_{x}(s, X(s)) a(s) d B_{H}(s)
$$

\footnotetext{
${ }^{2}$ See $\mathrm{Hu}$ and $\varnothing \mathrm{ksendal}[9]$ for more on this
} 


$$
+\int_{0}^{t} f_{x x}(s, X(s))\left\{a(s) \int_{0}^{s} \phi(s, u) a(u) d u\right\} d s
$$

We shall in connection with Asian options need a minor generalization of this formula. In Biagini and $\varnothing \mathrm{ksendal}[2]$ they present a general multidimensional version of the fractional Itô Formula. We will here restrict ourselves to a corollary of their result, avoiding a lot of technicalities and notation which is unnecessary for our purposes.

Corollary 2.4. Assume $X(t)=\int_{0}^{t} a(s) d B_{H}(s)$ and $Y(t)=\int_{0}^{t} b(s) d B_{H}(s)$, where $a, b \in$ $L_{\phi}^{2}(\mathbb{R})$. For a function $f \in C^{1,2}\left(\mathbb{R}_{+} \times \mathbb{R}^{2}\right)$ with bounded derivatives we have

$$
\begin{aligned}
f(t, X(t), & Y(t))=f(0,0,0)+\int_{0}^{t} f_{t}(s, X(s), Y(s)) d s \\
& +\int_{0}^{t} f_{x}(s, X(s), Y(s)) a(s)+f_{y}(s, X(s), Y(s)) b(s) d B_{H}(s) \\
& +\int_{0}^{t} f_{x x}(s, X(s), Y(s))\left\{a(s) \int_{0}^{s} \phi(s, u) a(u) d u\right\} d s \\
& +\int_{0}^{t} f_{x y}(s, X(s), Y(s))\left\{a(s) \int_{0}^{s} \phi(s, u) b(u) d u+b(s) \int_{0}^{s} \phi(s, u) a(u) d u\right\} \\
& +\int_{0}^{t} f_{y y}(s, X(s), Y(s))\left\{b(s) \int_{0}^{s} \phi(s, u) b(u) d u\right\} d s
\end{aligned}
$$

Proof. This is a simple consequence of Thm. 2.6 in Biagini and Øksendal [2].

Motivated from the white noise analysis, we define the quasi-conditional expectation of a random variable in $L^{2}(P)$ :

Definition 2.2. Let $X \in L^{2}(P)$ have chaos expansion $X=\sum_{n=0}^{\infty} I_{n}\left(f_{n}\right)$. The quasiconditional expectation of $X$ with respect to $\mathcal{F}_{t}^{H}$ is defined as

$$
\widehat{\mathrm{E}}\left[X \mid \mathcal{F}_{t}^{H}\right]=\sum_{n=0}^{\infty} I_{n}\left(f_{n} 1_{[0, t]}^{\otimes n}\right) .
$$

Observe that

$$
\widehat{\mathrm{E}}\left[X \mid \mathcal{F}_{0}^{H}\right]=f_{0}=\mathrm{E}[X]
$$

It is also relativly easy to see that if $X$ is $\mathcal{F}_{t}^{H}$-adapted, it holds

$$
\widehat{\mathrm{E}}\left[X \mid \mathcal{F}_{t}^{H}\right]=X \text {. }
$$

The quasi-conditional expectation is a norm-contracting operator on $L^{2}(P)$ :

Lemma 2.5. For $X \in L^{2}(P)$ we have

$$
E\left[\left(\widehat{E}\left[X \mid \mathcal{F}_{t}^{H}\right]\right)^{2}\right] \leq E\left[X^{2}\right]
$$


Proof. Let the chaos expansion of $X$ be $X=\sum_{n=0}^{\infty} I_{n}\left(f_{n}\right)$. By the chaos expansion theorem and the definition of quasi-conditional expectation,

$$
\mathrm{E}\left[\left(\widehat{\mathrm{E}}\left[X \mid \mathcal{F}_{t}^{H}\right]\right)^{2}\right]=\sum_{n=0}^{\infty} n !\left|f_{n} 1_{[0, t]}^{\otimes n}\right|_{\phi, n}^{2} \leq \sum_{n=0}^{\infty} n !\left|f_{n}\right|_{\phi, n}^{2}=\mathrm{E}\left[X^{2}\right] .
$$

In the estimation we used that $\left|f 1_{A}\right|_{\phi, n} \leq|f|_{\phi, n}$ for $A \subset \mathbb{R}^{n}$ a Borel set and $f \in L_{\phi}^{2}\left(\mathbb{R}^{n}\right)$. This proves the assertion.

It follows directly from this Lemma that the quasi-conditional expectation is a random variable in $L^{2}(P)$. Moreover, we have the following convergence result:

Lemma 2.6. Assume $X_{n} \rightarrow X$ in $L^{2}(P)$. Then

$$
\widehat{E}\left[X_{n} \mid \mathcal{F}_{t}^{H}\right] \rightarrow \widehat{E}\left[X \mid \mathcal{F}_{t}^{H}\right]
$$

in $L^{2}(P)$.

Proof. The proof goes by a direct application of the norm-contracting property of the quasi-conditional expectation.

The quasi-conditional expectation does not coincide with the classical conditional expectation except for the case when $H=0.5$, that is, in the case of Brownian motion. Furthermore, we have that

$$
\widehat{\mathrm{E}}\left[\widehat{\mathrm{E}}\left[X \mid \mathcal{F}_{t}^{H}\right]\right]=\mathrm{E}[X]
$$

This property is called the law of double expectation, which is a well-known property for classical conditional expectation. From the definition of quasi-conditional expectation we define a martingale-type property, called the quasi-martingale property which was first introduced by $\mathrm{Hu}$ and Øksendal [9].

Definition 2.3. Assume $M(t) \in L^{2}(P)$ is an $\mathcal{F}_{t}^{H}$-adapted stochastic process. $M(t)$ is called a quasi-martingale if

$$
\widehat{\mathrm{E}}\left[M(t) \mid \mathcal{F}_{s}^{H}\right]=M(s)
$$

for every $0 \leq s \leq t<\infty$.

Following the arguments in Hida [6, Thm 4.6], we can characterize the chaos kernels of a quasi-martingale. If $M(t) \in L^{2}(P)$ is a quasi-martingale, then

$$
M(t)=\sum_{n=0}^{\infty} I_{n}\left(f_{n} 1_{[0, t]}^{\otimes n}\right)
$$

where $f_{n} \in L_{\phi, l o c}^{2}(\mathbb{R})$.

As will be important later, we note that the quasi-conditional expectation itself defines a quasi-martingale: By the law of double expectation, the process $M(t):=\widehat{\mathrm{E}}\left[X \mid \mathcal{F}_{t}^{H}\right]$ is a quasi-martingale whenever $X \in L^{2}(P)$. We derive a quasi-martingale representation theorem: 
Proposition 2.7. If $M(t)$ is a quasi-martingale with chaos expansion $M(t)=\sum_{n=0}^{\infty} I_{n}\left(f_{n} 1_{[0, t]}^{\otimes n}\right)$, then

$$
M(t)=M(0)+\int_{0}^{t} N(s) d B_{H}(s)
$$

where

$$
N(t)=\sum_{n=0}^{\infty} I_{n}\left((n+1) f_{n+1}(t, \cdot) 1_{[0, t)}^{\otimes n}\right) .
$$

Proof. Consider $N(t)=\sum_{n=0}^{\infty} I_{n}\left((n+1) 1_{[0, t)}^{\otimes n} f_{n+1}(t, \cdot)\right)$. From the definition of fractional Itô integration, we find

$$
\begin{aligned}
\int_{0}^{t} N(s) d B_{H}(s) & =\sum_{n=0}^{\infty} I_{n+1}\left(\frac{1}{n+1}(n+1) 1_{[0, t)}^{\otimes n} \widehat{f_{n+1}}(\cdot, \cdot)\right) \\
& =\sum_{n=0}^{\infty} I_{n+1}\left(1_{[0, t)}^{\otimes(n+1)} f_{n+1}\right)=\sum_{n=1}^{\infty} I_{n}\left(1_{[0, t]}^{\otimes n} f_{n}\right) \\
& =M(t)-M(0) .
\end{aligned}
$$

This proves the Proposition.

It is straightforward to see that fractional Brownian motion is a quasi-martingale. Moreover, the stochastic exponential with $a \in L_{\phi}^{2}(\mathbb{R})$,

$$
Y(t)=\exp \left(\int_{0}^{t} a(s) d B_{H}(s)-\frac{1}{2}\left|a 1_{[0, t]}\right|_{\phi}^{2}\right),
$$

is a quasi-martingale, too. There are two ways to see this: First, the fractional Itô Formula yields

$$
Y(t)=1+\int_{0}^{t} a(s) Y(s) d B_{H}(s),
$$

which defines a quasi-martingale by the quasi-martingale representation theorem. Secondly, a general stochastic exponential

$$
Y=\exp \left(\int_{\mathbb{R}} b(s) d B_{H}(s)-\frac{1}{2}|b|_{\phi}^{2}\right),
$$

for $b \in L_{\phi}^{2}(\mathbb{R})$ can be written as a Wick exponential

$$
Y=\exp ^{\diamond}\left(\int_{\mathbb{R}} b(s) d B_{H}(s)\right):=\sum_{n=0}^{\infty} I_{n}\left(\frac{1}{n !} b^{\otimes n}\right) .
$$

The quasi-conditional expectation of the Wick exponential is by definition

$$
\begin{aligned}
\widehat{\mathrm{E}}\left[\exp ^{\diamond}\left(\int_{\mathbb{R}} b(s) d B_{H}(s)\right) \mid \mathcal{F}_{t}^{H}\right] & =\exp ^{\diamond}\left(\int_{0}^{t} b(s) d B_{H}(s)\right) \\
& =\exp \left(\int_{0}^{t} b(s) d B_{H}(s)-\frac{1}{2}\left|b 1_{[0, t]}\right|_{\phi}^{2}\right) .
\end{aligned}
$$


From these calculations it is easily seen that $Y(t)$ is a quasi-martingale. This result will be useful in deriving price dynamics for temperature derivatives.

We say that a stochastic process $S(t) \in L^{2}(P)$ is a quasi-semimartingale if it exist $\mathcal{F}_{t}^{H}$-adapted stochastic processes $Y(t), Z(t) \in L^{2}(P)$ such that

$$
S(t)=S(0)+\int_{0}^{t} Y(s) d s+\int_{0}^{t} Z(s) d B_{H}(s) .
$$

A quasi-semimartingale is a process which can be decomposed into a quasi-martingale and a process of finite variation. Note that $f\left(t, B_{H}(t)\right)$ becomes a quasi-semimartingale by appealing to the fractional Itô Formula, as long as $f \in C^{1,2}\left(\mathbb{R}_{+} \times \mathbb{R}\right)$ with bounded derivatives.

We end this section with a fractional version of the Girsanov transform. This result will later be used to generate arbitrage-free price dynamics of weather derivatives. The following formulation is taken from $\mathrm{Hu}$ and Øksendal [9]:

Theorem 2.8. Let $\theta, \Theta$ be measurable functions with support on $[0, T]$, where $\theta$ is continuous and $\Theta$ is the solution of the integral equation $\int_{0}^{T} \Theta(s) \phi(s, t) d s=\theta(t)$ for $0 \leq t \leq T$. Then

$$
\widetilde{B}_{H}(t)=B_{H}(t)+\int_{0}^{t} \theta(s) d s
$$

is a fractional Brownian motion under the probability measure $Q$ on $\left(\Omega, \mathcal{F}_{T}^{H}\right)$, which is equivalent with $P$ and

$$
\frac{d Q}{d P}=\exp \left(-\int_{0}^{T} \Theta(s) d B_{H}(s)-\frac{1}{2}\left|\Theta 1_{[0, T]}\right|_{\phi}^{2}\right)
$$

being the Radon-Nikodym derivative.

\section{Dynamics For the temperature And its AVERAGE}

We introduce a dynamics for the temperature based on fractional Brownian motion. The dynamics will be of Ornstein-Uhlenbeck type, and we consider time-dependent coefficients to allow for seasonal variation. Such a model was first introduced and analyzed in Brody, Syroka and Zervos [3]. Since we are also interested in Asian options, we state a dynamics for the average temperature derived from the fractional Ornstein-Uhlenbeck model for temperature.

Let the daily temperature follow the (fractional) stochastic differential equation

$$
X(t)=x+\int_{0}^{t} \alpha(s)(\lambda(s)-X(s)) d s+\int_{0}^{t} \sigma(s) d B_{H}(s),
$$

where $\lambda(t)$ is the seasonal trend, $\alpha(t)>0$ the speed of mean-reversion towards this trend, and $\sigma(t)>0$ the volatility. We assume $\lambda$ to be a measurable and bounded function, $\alpha \in C(\mathbb{R})$ and $\sigma \in L_{\phi}^{2}(\mathbb{R}) \cap C(\mathbb{R})$. An explicit solution of the stochastic differential equation 
for $X(t)$ can be found by a straightforward application of the fractional Itô Formula:

$$
X(t)=\mathrm{e}^{-\int_{0}^{t} \alpha(s) d s}\left(x+\int_{0}^{t} \alpha(s) \lambda(s) \mathrm{e}^{\int_{0}^{s} \alpha(u) d u} d s\right)+\int_{0}^{t} \sigma(s) \mathrm{e}^{-\int_{s}^{t} \alpha(u) d u} d B_{H}(s) .
$$

Brody, Syroka and Zervos [3] estimated the Hurst exponent to be $H=0.61$ for the daily central England temperature (CET). Their estimate was based on a data set ranging from year 1772 up to 1999, demonstrating that temperature is significantly persistent and henceforth that fractional Brownian motion is a reasonable model for the random fluctuations.

Consider the Girsanov transform

$$
\widetilde{B}_{H}(t)=\int_{0}^{t} \frac{\alpha(s)}{\sigma(s)} \theta(s) d s+B_{H}(t)
$$

where $\theta$ is a continuous function with support in $[0, T]$ such that there exists a function $\Theta$ with suppport in $[0, T]$ and solving the integral equation $\int_{0}^{T} \Theta(s) \phi(s, t) d s=\alpha(t) \theta(t) / \sigma(t)$ for all $0 \leq t \leq T$. Denote by $Q$ the probability measure under which $\widetilde{B}_{H}$ is a fractional Brownian motion. We see that the dynamics of $X$ under $Q$ is given by

$$
X(t)=x+\int_{0}^{t} \alpha(s)(\lambda(s)+\theta(s)-X(s)) d s+\int_{0}^{t} \sigma(s) d \widetilde{B}_{H}(s) .
$$

The explicit dynamics of the temperature under $Q$ will be, using the same argumentation as above,

$$
X(t)=\mathrm{e}^{-\int_{0}^{t} \alpha(s) d s}\left(x+\int_{0}^{t} \alpha(s)(\lambda(s)+\theta(s)) \mathrm{e}^{\int_{0}^{s} \alpha(u) d u} d s\right)+\int_{0}^{t} \sigma(s) \mathrm{e}^{-\int_{s}^{t} \alpha(u) d u} d \widetilde{B}_{H}(s) .
$$

The function $\theta$ is the market price of risk and the dynamics of $X(t)$ under $Q$ the risk-neutral dynamics.

Example 3.1. In incomplete financial markets it is not uncommon to specify the market price of risk as a constant $\theta(t) \equiv \theta$. If we assume that $\alpha$ and $\sigma$ are positive constants as well, we must search for a function $\Theta(t)$ such that $\int_{0}^{T} \Theta(t) \phi(s, t) d s=\alpha \theta / \sigma$ for all $0 \leq t \leq T$. In fact, Hu and Øksendal [9] have calculated this in a different context to be

$$
\Theta(t)=c\left(T t-t^{2}\right)^{0.5-H}
$$

for $0 \leq t \leq T$, and zero otherwise, where

$$
c=\frac{\alpha \theta}{2 \sigma H(2 H-1) \Gamma(2 H-1) \Gamma(2-2 H) \cos (\pi(H-1 / 2))} .
$$

Thus, we have specified completely the necessary ingredients in the Girsanov transform when the market price of risk, volatility and speed of mean reversion are constants. If $\alpha$ and/or $\sigma$ are time-dependent, we have an integral equation for $\Theta$ which can be studied in light of the theory of Lundgren and Chiang [13]. 
The average temperature over the time interval $[0, T]$ is

$$
\begin{aligned}
\int_{0}^{T} X(t) d t=\int_{0}^{T} \mathrm{e}^{-\int_{0}^{t} \alpha(s) d s} & \left(x+\int_{0}^{t} \alpha(s) \lambda(s) \mathrm{e}^{\int_{0}^{s} \alpha(u) d u} d s\right) d t \\
& +\int_{0}^{T} \int_{0}^{t} \sigma(s) \mathrm{e}^{-\int_{s}^{t} \alpha(u) d u} d B_{H}(s) d t .
\end{aligned}
$$

Admittedly, one should divide by $T$ in order to justify the term average. However, we have chosen to keep this name since there will be no essential difference when we reach Asian options. The fractional Itô integral can be rewritten as follows,

$$
\begin{aligned}
\int_{0}^{T} \int_{0}^{t} \sigma(s) \mathrm{e}^{-\int_{s}^{t} \alpha(u) d u} d B_{H}(s) d t & =\int_{0}^{T} \int_{0}^{T} \sigma(s) \mathrm{e}^{-\int_{s}^{t} \alpha(u) d u} 1_{[0, t]}(s) \diamond W_{H}(s) d s d t \\
& =\int_{0}^{T} \int_{0}^{T} 1_{[0, t]}(s) \mathrm{e}^{-\int_{s}^{t} \alpha(u) d u} d t \sigma(s) \diamond W_{H}(s) d s \\
& =\int_{0}^{T}\left(\int_{s}^{T} \mathrm{e}^{-\int_{s}^{t} \alpha(u) d u} d t\right) \sigma(s) d B_{H}(s) .
\end{aligned}
$$

In the second equality we used Fubini's theorem applied on Pettis integrals. Hence, the average temperature becomes

$$
\begin{aligned}
\int_{0}^{T} X(t) d t=\int_{0}^{T} \mathrm{e}^{-\int_{0}^{t} \alpha(s) d s} & \left(x+\int_{0}^{t} \alpha(s) \lambda(s) \mathrm{e}^{\int_{0}^{s} \alpha(u) d u} d s\right) d t \\
& +\int_{0}^{T}\left(\int_{s}^{T} \mathrm{e}^{-\int_{s}^{t} \alpha(u) d u} d t\right) \sigma(s) d B_{H}(s) .
\end{aligned}
$$

We find the risk-neutral dynamics of the average temperature by simply substituting $B_{H}$ with $\widetilde{B}_{H}$ and writing $\lambda+\theta$ instead of $\lambda$.

From above, we see that both temperature $X(t)$ and its average $\int_{0}^{T} X(t) d t$ are Gaussian random variables. This holds true both under $P$ and in the risk-neutral context since they both are represented as a deterministic function plus a fractional Itô integral with a deterministic integrand. The latter is known to be a Gaussian random variable with zero expectation (see $\mathrm{Hu}$ and Øksendal [9]).

\section{Arbitrage-Free Price Dynamics of Weather Derivatives}

We consider a market where the investor can speculate in a risk-free bond and a weather derivative on temperature. The bond is assumed to follow the dynamics

$$
d R(t)=r R(t) d t
$$

with $R(0)=1$ and $r$ being the continuously compounding risk-free interest rate. The derivative is assumed to be a contingent claim on temperature:

Definition 4.1. A random variable $Y \in L^{2}(P)$ is called a contingent claim on temperature if $Y$ is $\mathcal{F}_{T}^{H}$-measurable, $T$ being the "expiry" date of the contingent claim. 
Typical examples of contingent claims can be a plain vanilla European options on temperature or Asian type of options with the average temperature as underlying. The former has a general payoff function $f(X(T))$, while the latter has payoff $f\left(\int_{0}^{T} X(t) d t\right)$. We shall study the price dynamics of both types of derivatives in this paper.

Let $(a, b)$ be a portfolio strategy where $a(t)$ is the number of bonds and $b(t)$ the number of contingent claims $Y$ held at time $t$. Assume that $C(t)$ is the price of the claim at time $t$, and that $C(t)$ is a quasi-semimartingale. We say that the portfolio strategy $(a, b)$ is self-financing if

$$
V(t)=V(0)+\int_{0}^{t} a(s) d R(s)+\int_{0}^{t} b(s) d C(s)
$$

A self-financing portfolio will be a quasi-martingale after discounting under a suitable risk-neutral probability $Q$, a well-known result from the standard Brownian motion case:

Lemma 4.1. Let $(a, b)$ be a self-financing strategy. Then

$$
e^{-r t} V(t)=V(0)+\int_{0}^{t} b(s) d\left(e^{-r s} C(s)\right) .
$$

Proof. Let $C(t)$ have the following differential

$$
d C(t)=Y(t) d t+Z(t) d B_{H}(t) .
$$

Assume for simplicity that $Y(t)=0$. We calculate

$$
\begin{aligned}
\mathrm{e}^{-r t} C(t) & =C(0)+\int_{0}^{t} \frac{d}{d s}\left(\mathrm{e}^{-r s} \int_{0}^{s} Z(u) \diamond W^{H}(u) d u\right) d s \\
& =C(0)+\int_{0}^{t}\left(\mathrm{e}^{-r s} Z(s) \diamond W^{H}(s)-r \mathrm{e}^{-r s} \int_{0}^{s} Z(u) d B_{H}(u)\right) d s \\
& =C(0)+\int_{0}^{t} \mathrm{e}^{-r s} d C(s)-r \int_{0}^{t} \mathrm{e}^{-r s} C(s) d s
\end{aligned}
$$

Using a similar argument for $\mathrm{e}^{-r t} V(t)$, we find for the self-financing portfolio:

$$
\begin{aligned}
\mathrm{e}^{-r t} V(t) & =V(0)+\int_{0}^{t} \mathrm{e}^{-r s} d V(s)-r \int_{0}^{t} \mathrm{e}^{-r s} V(s) d s \\
& =V(0)+\int_{0}^{t} \mathrm{e}^{-r s} a(s) d R(s)+\int_{0}^{t} \mathrm{e}^{-r s} b(s) d C(s)-r \int_{0}^{t} \mathrm{e}^{-r s} V(s) d s \\
& =V(0)+r \int_{0}^{t} \mathrm{e}^{-r s}(V(s)-b(s) C(s)) d s+\int_{0}^{t} b(s) \mathrm{e}^{-r s} d C(s)-r \int_{0}^{t} \mathrm{e}^{-r s} V(s) d s \\
& =V(0)+\int_{0}^{t} b(s)\left(\mathrm{e}^{-r s} d C(s)-r \mathrm{e}^{-r s} C(s) d s\right) \\
& =V(0)+\int_{0}^{t} b(s) d\left(\mathrm{e}^{-r s} C(s)\right)
\end{aligned}
$$

This yields the Lemma. 
Note that by the quasi-martingale representation theorem the right-hand side of (4.2) is interpreted as a fractional Itô integral whenever the discounted price of the claim is a quasi-martingale (under some risk neutral measure). Note the parallel to the theory for Brownian motion.

We recall the standard definition of an arbitrage opportunity (or simply arbitrage): A self-financing strategy $(a, b)$ is an arbitrage if $V(0) \leq 0, V(t) \geq 0$ a.s. and $V(t)>0$ with strictly positive probability.

Definition 4.2. A price process $C(t)$ of $Y$ is called arbitrage-free if there exist no selffinancing portfolio strategy which is an arbitrage.

It is now straightforward to see that any quasi-martingale dynamics for $C(t)$ will imply an arbitrage-free dynamics, as in classical theory for incomplete markets. The main result of this paper is the following theorem, which states a class of arbitrage-free price dynamics for a contingent claim $Y$ on temperature:

Theorem 4.2. Let $Y$ be a contingent claim on temperature. Assume $Q$ is an equivalent probability measure of $P$ given by a Girsanov transform and $Y \in L^{2}(Q)$. An arbitrage-free price $C(t)$ of $Y$ at time $t \in[0, T]$ is given by

$$
C(t)=e^{-r(T-t)} \widehat{E}_{Q}\left[Y \mid \mathcal{F}_{t}^{H}\right],
$$

where $\widehat{E}_{Q}$ is the (quasi-conditional) expectation under the probability $Q$.

Proof. Denote by $M(t)=\mathrm{e}^{-r T} \widehat{\mathrm{E}}_{Q}\left[Y \mid \mathcal{F}_{t}^{H}\right]$. Then $M(t)$ is a quasi-martingale and $M(t)=$ $\mathrm{e}^{-r t} C(t)$. Let $a(t)$ be the holdings in bonds and $b(t)$ the number of weather claims held by an investor at time $t$, and assume that the strategy is self-financing. The value of the portfolio at time $t$ will be $V(t)=a(t) R(t)+b(t) C(t)$. Discounting by $r$ and appealing to the self-financing hypothesis yield

$$
\mathrm{e}^{-r t} V(t)=V(0)+\int_{0}^{t} b(s) d M(s) .
$$

Hence, $\mathrm{e}^{-r t} V(t)$ is a fractional quasi-martingale under the probability $Q$. We therefore observe that there are no arbitrage possibilites, since whenever $V(0) \leq 0$ the fractional quasi-martingale property of $\mathrm{e}^{-r t} V(t)$ yields that $\mathrm{E}_{Q}[V(t)] \leq 0$, and thus $P(V(t)<0)>0$, since $Q$ is equivalent with $P$. We can conclude that $C(t)$ is an arbitrage-free price dynamics of $Y$.

In the Theorem above, we need to assume that $Q$ comes from a Girsanov transform of $P$ in order to have a new fractional Brownian motion which defines the quasi-conditional expectation. If we parametrize the Girsanov transform as in (3.3), we know the risk-neutral dynamics of the temperature process, and can perform calculations of the price dynamics including a market price of risk. This will provide us with theoretical price dynamics, one for each choice of $\theta$, which can be calibrated to observed market prices of temperature claims, thus fixing the market price of risk. $Y$.

Observe that $C(0)=\mathrm{e}^{-r T} \mathrm{E}_{Q}[Y]$ is the price when entering the contract, and that $C(T)=$ 


\section{A Fourier approach to the Dynamic PRICING of Claims on temperature}

We first state a general Proposition which shows how one can calculate the quasiconditional expectation involved in the pricing dynamics of temperature claims. The Proposition is generalizing a similar result in Necula [14]:

Proposition 5.1. Let $b \in L_{\phi}^{2}(\mathbb{R})$, and define the function

$$
p_{t, T}(x)=\frac{1}{\sqrt{2 \pi \Phi(t, T)}} \exp \left(-x^{2} / 2 \Phi(t, T)\right)
$$

where $\Phi(t, T)=\left|b 1_{[0, T]}\right|_{\phi}^{2}-\left|b 1_{[0, t]}\right|_{\phi}^{2}$. Assume $f \in L^{2}\left(\mathbb{R}, p_{0, T}(x) d x\right)$, then

$$
\widehat{E}\left[f\left(\int_{0}^{T} b(s) d B_{H}(s)\right) \mid \mathcal{F}_{t}^{H}\right]=\int_{\mathbb{R}} f(y) p_{t, T}\left(\int_{0}^{t} b(s) d B_{H}(s)-y\right) d y .
$$

Proof. Observe that the assumption $f \in L^{2}\left(\mathbb{R}, p_{0, T}(x) d x\right)$ implies $f\left(\int_{0}^{T} b(s) d B_{H}(s)\right) \in$ $L^{2}(P)$, since $p_{0, T}(x)$ is the probability density of $\int_{0}^{T} b(s) d B_{H}(s)$. In the first part of the proof, we establish the result when $f$ and its Fourier transform $\hat{f}$ are elements of $L^{1}(\mathbb{R})$. The general case follows by an approximation argument relying on the contraction property of quasi-conditional expectation.

Assume $f$ and its Fourier transform $\hat{f}$ are elements of $L^{1}(\mathbb{R})$ : From the defintion of the Fourier transform and its inverse we have

$$
f(x)=\frac{1}{2 \pi} \int_{\mathbb{R}} \hat{f}(\xi) \exp (i \xi x) d \xi
$$

We now calculate using the rules of the quasi-conditional expectation:

$$
\begin{aligned}
\widehat{\mathrm{E}}\left[f\left(\int_{0}^{T} b(s) d B_{H}(s)\right) \mid \mathcal{F}_{t}^{H}\right] \\
\quad=\frac{1}{2 \pi} \int_{\mathbb{R}} \hat{f}(\xi) \widehat{\mathrm{E}}\left[\exp \left(i \xi \int_{0}^{T} b(s) d B_{H}(s)\right) \mid \mathcal{F}_{t}^{H}\right] d \xi \\
=\frac{1}{2 \pi} \int_{\mathbb{R}} \hat{f}(\xi) \exp \left(-\frac{1}{2} \xi^{2}\left|b 1_{[0, T]}\right|_{\phi}^{2}\right) \widehat{\mathrm{E}}\left[\exp ^{\diamond}\left(i \xi \int_{0}^{T} b(s) d B_{H}(s)\right) \mid \mathcal{F}_{t}^{H}\right] d \xi \\
=\frac{1}{2 \pi} \int_{\mathbb{R}} \hat{f}(\xi) \exp \left(-\frac{1}{2} \xi^{2}\left|b 1_{[0, T]}\right|_{\phi}^{2}\right) \widehat{\mathrm{E}}\left[\exp ^{\diamond}\left(i \xi \int_{0}^{t} b(s) d B_{H}(s)\right)\right] d \xi \\
=\frac{1}{2 \pi} \int_{\mathbb{R}} \hat{f}(\xi) \exp \left(-\frac{1}{2} \xi^{2} \Phi(t, T)\right) \exp \left(i \xi \int_{0}^{t} b(s) d B_{H}(s)\right) d \xi \\
=\int_{\mathbb{R}} f(y) p_{t, T}\left(\int_{0}^{t} b(s) d B_{H}(s)-y\right) d y .
\end{aligned}
$$

The last equality follows from the Fourier inversion formula and the fact that the Fourier transform of $p_{t, T}(x)$ is equal to $\exp \left(-\frac{1}{2} \xi^{2} \Phi(t, T)\right)$. This concludes the proof when $f$ and its Fourier transform are elements of $L^{1}(\mathbb{R})$. 
To simplify the rest of the argument, we assume without loss of generality that $b=1$. It is trivially seen that $f\left(B_{H}(T)\right) \in L^{2}(P)$ when $f \in L^{2}\left(\mathbb{R}, p_{0, T}(x) d x\right)$, since $p_{0, T}(x)$ is the density function of $B_{H}(T)$. By Prop. 7.9 in Folland [5], the space $C_{c}(\mathbb{R})$ is dense in $L^{2}\left(\mathbb{R}, p_{0, T}(x) d x\right)$, and thus also $C_{c}^{\infty}(\mathbb{R})$, the space of infinitely continuously differentiable functions with compact support, since this space is dense in the uniform norm in $C_{c}(\mathbb{R})$. We can therefore find a sequence of functions $\left\{f_{n}\right\} \subset C_{c}^{\infty}(\mathbb{R})$ such that $f_{n} \rightarrow f$ in $L^{2}\left(\mathbb{R}, p_{0, T}(x) d x\right)$. From the relation

$$
\mathrm{E}\left[\left|f_{n}\left(B_{H}(T)\right)-f\left(B_{H}(T)\right)\right|^{2}\right]=\int_{\mathbb{R}}\left|f_{n}(y)-f(y)\right|^{2} p_{0, T}(y) d y \rightarrow 0
$$

we see that $f_{n}\left(B_{H}(T)\right) \rightarrow f\left(B_{H}(T)\right)$ in $L^{2}(P)$. By Lemma 2.6, it follows that

$$
\widehat{\mathrm{E}}\left[f_{n}\left(B_{H}(T)\right) \mid \mathcal{F}_{t}^{H}\right] \rightarrow \widehat{\mathrm{E}}\left[f\left(B_{H}(T)\right) \mid \mathcal{F}_{t}^{H}\right]
$$

in $L^{2}(P)$. But, since $f_{n} \in C_{c}^{\infty}(\mathbb{R})$, which is a subset of the tempered Schwartz functions $\mathcal{S}(\mathbb{R})$, we have that $\hat{f}_{n} \in \mathcal{S}(\mathbb{R}) \subset L^{1}(\mathbb{R})$, because the Fourier transform is closed in this function space (see e.g. Folland [5]). Hence, from the first part of the proof,

$$
\begin{aligned}
\widehat{\mathrm{E}}\left[f_{n}\left(B_{H}(T)\right) \mid \mathcal{F}_{t}^{H}\right] & =\int_{\mathbb{R}} f_{n}(y) p_{t, T}\left(B_{H}(t)-y\right) d y \\
& =\int_{\mathbb{R}} f_{n}\left(B_{H}(t)-y\right) p_{t, T}(y) d y
\end{aligned}
$$

We can therefore conclude

$$
\widehat{\mathrm{E}}\left[f\left(B_{H}(T)\right) \mid \mathcal{F}_{t}^{H}\right]=L^{2}(P)-\lim _{n \rightarrow \infty} \int_{\mathbb{R}} f_{n}(y) p_{t, T}\left(B_{H}(t)-y\right) d y .
$$

In order to finalize the argument, we need to show that $\int_{\mathbb{R}} f(y) p_{t, T}\left(B_{H}(t)-y\right) d y \in L^{2}(P)$ and that the right-hand side of (5.2) is equal to this expression:

By the Minkowski and Cauchy-Schwartz inequalities we find,

$$
\begin{aligned}
\mathrm{E}\left[\left(\int_{\mathbb{R}} f\left(B_{H}(t)-y\right) p_{t, T}(y) d y\right)^{2}\right]^{1 / 2} & \leq \int_{\mathbb{R}} \mathrm{E}\left[f^{2}\left(B_{H}(t)-y\right)\right]^{1 / 2} p_{t, T}(y) d y \\
& =\int_{\mathbb{R}} \mathrm{E}\left[f^{2}\left(B_{H}(t)-y\right)\right]^{1 / 2} p_{t, T}^{1 / 2}(y) p_{t, T}^{1 / 2}(y) d y \\
& \leq\left(\int_{\mathbb{R}} \mathrm{E}\left[f^{2}\left(B_{H}(t)-y\right)\right] p_{t, T}(y) d y\right)^{1 / 2}\left(\int_{\mathbb{R}} p_{t, T}(y) d y\right)^{1 / 2} \\
& =\left(\int_{\mathbb{R}} \int_{\mathbb{R}} f^{2}(x-y) p_{0, t}(x) d x p_{t, T}(y) d y\right)^{1 / 2} \\
& =\left(\int_{\mathbb{R}} f^{2}(y) \int_{\mathbb{R}} p_{0, t}(x) p_{t, T}(x-y) d x d y\right)^{1 / 2} \\
& =\left(\int_{\mathbb{R}} f^{2}(y)\left(p_{0, t} \star p_{t, T}\right)(y) d y\right)^{1 / 2}
\end{aligned}
$$


From the semi-group property of $p_{0, t}$ (or, by appealing to the Fourier transform), it holds $p_{0, t} \star p_{t, T}=p_{0, T}$. Therefore,

$$
\mathrm{E}\left[\left(\int_{\mathbb{R}} f\left(B_{H}(t)-y\right) p_{t, T}(y) d y\right)^{2}\right]^{1 / 2}=\left(\int_{\mathbb{R}} f^{2}(y) p_{0, T}(y) d y\right)^{1 / 2}<\infty .
$$

Hence, $\int_{\mathbb{R}} f(y) p_{t, T}\left(B_{H}(t)-y\right) d y \in L^{2}(P)$. By a similar argument we find

$\mathrm{E}\left[\left(\int_{\mathbb{R}}\left(f_{n}\left(B_{H}(t)-y\right)-f\left(B_{H}(t)-y\right)\right) p_{t, T}(y) d y\right)^{2}\right]^{1 / 2}=\left(\int_{\mathbb{R}}\left(f(y)-f_{n}(y)\right)^{2} p_{T}(y) d y\right)^{1 / 2} \rightarrow 0$.

This completes the proof of the Proposition.

Formulas for the arbitrage-free price dynamics of a European and Asian claim on temperature follow easily from the Proposition. Consider first a European claim with payoff function $f(X(T))$. Appealing to the risk neutral dynamics of $X(t)$ given in (3.5) for a specified market price of risk $\theta(t)$, we define,

$$
\begin{aligned}
a_{T} & =\mathrm{e}^{-\int_{0}^{T} \alpha(s) d s}\left(x+\int_{0}^{T} \alpha(s)(\lambda(s)+\theta(s)) \mathrm{e}^{\int_{0}^{s} \alpha(u) d u} d s\right) \\
b_{T}(s) & =\sigma(s) \mathrm{e}^{-\int_{s}^{T} \alpha(u) d u} .
\end{aligned}
$$

Assuming $f\left(a_{T}+\cdot\right) \in L^{2}\left(\mathbb{R}, p_{0, T}(x) d x\right)$, where $p_{t, T}$ is given in Proposition 5.1 with $b_{T}$ as above, the arbitrage-free pricing dynamics (under $Q$ ) for the claim becomes

$$
C_{\text {europ }}^{\theta}(t)=\mathrm{e}^{-r(T-t)} \int_{\mathbb{R}} f(y) p_{t, T}\left(a_{T}+\int_{0}^{t} b_{T}(s) d \widetilde{B}_{H}(s)-y\right) d y .
$$

By manipulating with the expression (3.5), one easily derives that

$$
a_{T}+\int_{0}^{t} b_{T}(s) d \widetilde{B}_{H}(s)=c(t, T)+\mathrm{e}^{-\int_{t}^{T} \alpha(s) d s} X(t)
$$

with

$$
c(t, T)=\mathrm{e}^{-\int_{0}^{T} \alpha(s) d s} \int_{t}^{T} \alpha(s)(\lambda(s)+\theta(s)) \mathrm{e}^{\int_{0}^{s} \alpha(u) d u} d s .
$$

Hence, we obtain the price dynamics

$$
C_{\text {europ }}^{\theta}(t)=\mathrm{e}^{-r(T-t)} \int_{\mathbb{R}} f(y) p_{t, T}\left(c(t, T)+\mathrm{e}^{-\int_{t}^{T} \alpha(s) d s} X(t)-y\right) d y .
$$

Note that we can represent the price at time $t$ as a function of $t, T$ and $X(t)$, the current temperature. Introducing the function

$$
v_{\text {europ }}^{\theta}(t, T, x)=\mathrm{e}^{-r(T-t)} \int_{\mathbb{R}} f(y) p_{t, T}\left(c(t, T)+\mathrm{e}^{-\int_{t}^{T} \alpha(s) d s} x-y\right) d y,
$$

we have $C_{\text {europ }}^{\theta}(t)=v_{\text {europ }}^{\theta}(t, T, X(t))$. Since $p_{t, T}$ is a heat kernel, there is a close relationship to a backward parabolic equation of Black \& Scholes type which $v_{\text {europ }}^{\theta}$ solves. We will demonstrate this in the next Section. 

Q)

For an Asian claim with payoff function $f\left(\int_{0}^{T} X(t) d t\right)$ we set (taken from (3.6) under

$$
\begin{aligned}
a_{T} & =\int_{0}^{T} \mathrm{e}^{-\int_{0}^{s} \alpha(u) d u}\left(x+\int_{0}^{s} \alpha(v)(\lambda(v)+\theta(v)) \mathrm{e}^{\int_{0}^{v} \alpha(u) d u} d v\right) d s \\
b_{T}(s) & =\sigma(s) \int_{s}^{T} \mathrm{e}^{-\int_{s}^{v} \alpha(u) d u} d v .
\end{aligned}
$$

Assuming $f\left(a_{T}+\cdot\right) \in L^{2}\left(\mathbb{R}, p_{0, T}(x) d x\right)$, where $p_{t, T}$ is given in Proposition 5.1 with $b_{T}$ as above, the arbitrage-free pricing dynamics (under $Q$ ) for the Asian claim is

$$
C_{\text {asian }}^{\theta}(t)=\mathrm{e}^{-r(T-t)} \int_{\mathbb{R}} f(z) p_{t, T}\left(a_{T}+\int_{0}^{t} b_{T}(s) d \widetilde{B}_{H}(s)-z\right) d z .
$$

By manipulating with the risk-neutral version of (3.6) and (3.5) we find

$$
\begin{aligned}
& a_{T}+\int_{0}^{t} b_{T}(s) d \widetilde{B}_{H}(s)=a_{T}+\int_{0}^{t} \int_{s}^{T} \mathrm{e}^{-\int_{0}^{u} \alpha(v) d v} \mathrm{e}^{\int_{0}^{s} \alpha(u) d u} \sigma(s) d \widetilde{B}_{H}(s) \\
& =a_{T}+\int_{0}^{t} \int_{s}^{t} \mathrm{e}^{-\int_{0}^{u} \alpha(v) d v} \mathrm{e}^{\int_{0}^{s} \alpha(u) d u} \sigma(s) d \widetilde{B}_{H}(s) \\
& +\int_{t}^{T} \mathrm{e}^{-\int_{0}^{u} \alpha(v) d v} \cdot \int_{0}^{t} \mathrm{e}^{\int_{0}^{s} \alpha(u) d u} \sigma(s) d \widetilde{B}_{H}(s) \\
& =a_{T}+\int_{0}^{t} \int_{0}^{t} \mathrm{e}^{-\int_{s}^{u} \alpha(v) d v} d u \sigma(s) d \widetilde{B}_{H}(s) \\
& +\int_{t}^{T} \mathrm{e}^{\int_{u}^{t} \alpha(v) d v} d u \cdot \int_{0}^{t} \mathrm{e}^{-\int_{s}^{t} \alpha(v) d v} \sigma(s) d \widetilde{B}_{H}(s) \\
& =a_{T}+\left(\int_{0}^{t} X(s) d s-a_{t}\right)+\int_{t}^{T} \mathrm{e}^{\int_{u}^{t} \alpha(v) d v} d u \times \\
& \left(X(t)-\mathrm{e}^{-\int_{0}^{t} \alpha(v) d v}\left(x+\int_{0}^{t} \alpha(s)(\lambda(s)+\theta(s)) \mathrm{e}^{\int_{0}^{s} \alpha(v) d v} d s\right)\right) \\
& =d(t, T)+\int_{0}^{t} X(s) d s+X(t) \int_{t}^{T} \mathrm{e}^{\int_{u}^{t} \alpha(v) d v} d u,
\end{aligned}
$$

where

$$
d(t, T)=a_{T}-a_{t}-\int_{t}^{T} \mathrm{e}^{-\int_{0}^{u} \alpha(v) d v} d u\left(x+\int_{0}^{t} \alpha(s)(\lambda(s)+\theta(s)) \mathrm{e}^{\int_{0}^{s} \alpha(v) d v} d u\right) .
$$

Hence,

$$
C_{\text {asian }}^{\theta}(t)=\mathrm{e}^{-r(T-t)} \int_{\mathbb{R}} f(z) p_{t, T}\left(d(t, T)+\int_{0}^{t} X(s) d s+X(t) \int_{t}^{T} \mathrm{e}^{\int_{u}^{t} \alpha(v) d v} d u-z\right) d z
$$


For the Asian option the price can be represented as a function of $t, T, X(t)$, the current temperature, and $\int_{0}^{t} X(s) d s$, the average temperature up to the present time $t$, i.e.,

$$
C_{\text {asian }}^{\theta}(t)=v_{\text {asian }}^{\theta}\left(t, T, X(t), \int_{0}^{t} X(s) d s\right)
$$

where

$$
v_{\text {asian }}^{\theta}(t, T, x, y)=\mathrm{e}^{-r(T-t)} \int_{\mathbb{R}} f(z) p_{t, T}\left(d(t, T)+x \int_{t}^{T} \mathrm{e}^{\int_{u}^{t} \alpha(v) d v} d u+y-z\right) d z .
$$

Also in this case $v_{\text {asian }}^{\theta}$ will solve a backward parabolic equation of Black \& Scholes type.

Note that the payoff function for a plain vanilla call (put) option, $f(x)=\max (x-K, 0)$ $(f(x)=\max (K-x, 0))$, satisfies the integrability condition $f\left(a_{T}+\cdot\right) \in L^{2}\left(\mathbb{R}, p_{0, T}(x) d x\right)$. Recall that $p_{t, T}$ is given in Proposition 5.1 with $a_{T}$ and $b_{T}$ given as above for the European and Asian claim, respectivly.

The price of the claims will not, unlike in the case of Brownian motion, be a function of $T-t$, the time to expiration, but on $t$ and $T$ seperately. This property may be considered as undesirable from a market point of view from the following fact: Consider two plain vanilla call options both with expiry at time $T$ and strike $K$. The first is issued at time 0 , while the second is issued at some time $t \in(0, T)$. In the framework of Black \& Scholes these two contracts will have the same price at time $t$, otherwise a clear arbitrage opportunity exists. In economical terminology, the contracts will be perfect substitutes at time $t$. This is seemingly not the case in our context, and one may question the validity of the results above in a derivatives pricing context. However, if one wants to price temperature options with similar characteristics (like exercise time and payoff), but with different starting times, one needs to decide upon a point of origin for time which must be the same for all contracts and be used in the pricing instead of resetting the clock to zero when an option comes to life. In this way we avoid the arbitrage opportunity in the example above. It is a question, however, what should this starting origin be.

In the weather derivatives market a natural class of claims are call (or put) options on the accumulated Heating (Cooling) degree days over some time interval. This type of claims are studied in Brody, Syroka and Zervos [3], and fits into our framework as well. In fact, using the argumentation above we can easily obtain the formulas derived in Section 4 of their paper. We obtain the price dynamics over the whole lifespan of the contract.

We end this Section with a peculiar, but noteworthy consequence of Proposition 5.1. It turns out that the quasi-conditional expectation of fractional Brownian motion and the conditional (not quasi-!) expectation of a specific Itô integral coincide: Choosing $b=1$, we find from Proposition 5.1

$$
\widehat{\mathrm{E}}\left[f\left(B_{H}(T)\right) \mid \mathcal{F}_{t}^{H}\right]=v\left(t, T, B_{H}(t)\right)
$$

where

$$
v(t, T, x)=\int_{\mathbb{R}} f(y)\left(2 \pi\left(T^{2 H}-t^{2 H}\right)\right)^{-1 / 2} \exp \left(-(y-x)^{2} / 2\left(T^{2 H}-t^{2 H}\right)\right) d y .
$$


Now, calculate the following conditional expectation:

$$
\begin{aligned}
\mathrm{E}[f( & \left.\left.\sqrt{H} \int_{0}^{T} s^{H-1 / 2} d B(s)\right) \mid \mathcal{F}_{t}\right] \\
& =\frac{1}{2 \pi} \int_{\mathbb{R}} \hat{f}(\xi) \mathrm{E}\left[\exp \left(i \xi \sqrt{H} \int_{0}^{T} s^{H-1 / 2} d B(s)\right) \mid \mathcal{F}_{t}\right] d \xi \\
& =\frac{1}{2 \pi} \int_{\mathbb{R}} \hat{f}(\xi) \exp \left(-\frac{1}{2} \xi^{2} H \int_{0}^{T} s^{2 H-1} d s\right) \mathrm{E}\left[\exp ^{\diamond}\left(i \xi \sqrt{H} \int_{0}^{T} s^{H-1 / 2} d B(s)\right) \mid \mathcal{F}_{t}\right] d \xi \\
& =\frac{1}{2 \pi} \int_{\mathbb{R}} \hat{f}(\xi) \exp \left(-\frac{1}{2} \xi^{2}\left(T^{2 H}-t^{2 H}\right)\right) \exp \left(i \xi \sqrt{H} \int_{0}^{t} s^{H-1 / 2} d B(s)\right) d \xi \\
& =v\left(t, T, \sqrt{H} \int_{0}^{t} s^{H-1 / 2} d B(s)\right) .
\end{aligned}
$$

It is straightforward to see that $\sqrt{H} \int_{0}^{t} s^{H-1 / 2} d B(s)$ is a centered normal random variable with variance equal to $t^{2 H}$, thus being equal in distribution to fractional Brownian motion at each time instant $t$. We therefore have

$$
\mathrm{E}\left[f\left(\sqrt{H} \int_{0}^{T} s^{H-1 / 2} d B(s)\right) \mid \mathcal{F}_{t}\right]=\widehat{\mathrm{E}}\left[f\left(B_{H}(T)\right) \mid \mathcal{F}_{t}^{H}\right]
$$

where the equality is in distribution. Note however, that

$$
\mathrm{E}\left[\sqrt{H} \int_{0}^{t} u^{H-1 / 2} d B(u) \cdot \sqrt{H} \int_{0}^{s} u^{H-1 / 2} d B(u)\right]=(t \wedge s)^{2 H} \neq \mathrm{E}\left[B_{H}(t) B_{H}(s)\right] .
$$

The pathwise behaviour of the two conditional expectations are therefore different, and we expect them to be different in value, except at $t=0$, where they in fact will coincide.

\section{A fractional version of the Black \& Scholes partial differential EQUATION}

In this Section we use the fractional Itô Formula in order to identify solutions of backward parabolic equations as the quasi-conditional expectation of a functional of temperature. These results will connect the price dynamics of temperature claims to Black \& Scholes type partial differential equations.

Proposition 6.1. Let $X(t)$ be as in (3.5) and assume that $v \in C^{1,2}\left(\mathbb{R}_{+} \times \mathbb{R}\right)$ has bounded derivatives and solves the fractional Black and Scholes equation

$$
v_{t}(t, x)+\alpha(t)(\lambda(t)+\theta(t)-x) v_{x}(t, x)+\Psi(t) v_{x x}(t, x)=r v(t, x),
$$

on $(t, x) \in[0, T) \times \mathbb{R}$, with

$$
\Psi(t)=\sigma(t) e^{-\int_{0}^{t} \alpha(u) d u} \int_{0}^{t} \phi(t, s) \sigma(s) e^{-\int_{0}^{s} \alpha(u) d u} d s
$$


and terminal condition $v(T, x)=f(x)$. If $f(X(T)), v(t, X(t)) \in L^{2}(Q)$ for $t \in[0, T]$, where $Q$ is the equivalent martingale measure leading to (3.5), then

$$
v(t, X(t))=e^{-r(T-t)} \widehat{E}_{Q}\left[f(X(T)) \mid \mathcal{F}_{t}^{H}\right] .
$$

Proof. Assume without loss of generality that $r=0$. From (3.5) we can write $X(t)=$ $\mathrm{e}^{-\int_{0}^{t} \alpha(u) d u}(a(t)+Z(t))$ where

$$
a(t)=x+\int_{0}^{t} \alpha(s)(\lambda(s)+\theta(s)) \mathrm{e}^{\int_{0}^{s} \alpha(u) d u} d s,
$$

and $Z(t)=\int_{0}^{t} \sigma(s) \mathrm{e}^{\int_{0}^{s} \alpha(u) d u} d \widetilde{B}_{H}(s)$. Appealing to the fractional Itô Formula, we find

$$
\begin{aligned}
v(t, X(t))=v & (0, x)+\int_{0}^{t} v_{t}(s, X(s))+v_{x}(s, X(s)) \\
& \times\left(-\alpha(s) \mathrm{e}^{-\int_{0}^{t} \alpha(u) d u} a(s)+\mathrm{e}^{-\int_{0}^{s} \alpha(u) d u} \alpha(s)(\lambda(s)+\theta(s)) \mathrm{e}^{\int_{0}^{s} \alpha(u) d u}\right) d s \\
& +\int_{0}^{t} v_{x}(s, X(s)) \mathrm{e}^{-\int_{0}^{s} \alpha(u) d u} \sigma(s) \mathrm{e}^{\int_{0}^{s} \alpha(u) d u} d \widetilde{B}_{H}(s) \\
& +\int_{0}^{t} v_{x x}(s, X(s)) \mathrm{e}^{-2 \int_{0}^{s} \alpha(u) d u} \mathrm{e}^{\int_{0}^{s} \alpha(u) d u} \sigma(s) \int_{0}^{s} \phi(s, \tau) \sigma(\tau) \mathrm{e}^{\int_{0}^{\tau} \alpha(u) d u} d \tau d s \\
= & v(0, x)+\int_{0}^{t} \sigma(s) v_{x}(s, X(s)) d \widetilde{B}_{H}(s) .
\end{aligned}
$$

since $v$ solves the partial differential equation (6.1). Note that since $v_{x}$ is bounded and by the assumptions on integrability and smoothness of $\sigma$ we have that the fractional Itô integral on the right-hand side is in $L^{2}(Q)$, and thus a quasi-martingale. From the terminal condition we know that $v(T, X(T))=f(X(T))$. Hence,

$$
f(X(T))-v(t, x(t))=\int_{t}^{T} \sigma(s) v_{x}(s, X(s)) d \widetilde{B}_{H}(s) .
$$

From the assumption $f(X(T)), v(t, X(t)) \in L^{2}(Q)$, we find after taking quasi-conditional expectation on both sides and appealing to the $\mathcal{F}_{t}^{H}$-adaptedness of $v(t, X(t))$,

$$
\widehat{\mathrm{E}}_{Q}\left[f(X(T)) \mid \mathcal{F}_{t}^{H}\right]-v(t, X(t))=0
$$

This proves the Propostion.

We move on to Asian options, and arrive at the following result:

Proposition 6.2. Let $X(t)$ be as in (3.5) and assume that $v \in C^{1,2}\left(\mathbb{R}_{+} \times \mathbb{R}^{2}\right)$ has bounded derivatives and solves the fractional Black and Scholes equation

$v_{t}(t, x)+\alpha(t)(\lambda(t)+\theta(t)-x) v_{x}(t, x)+x v_{y}(t, x)+\Psi_{1}(t) v_{x x}(t, x)+\Psi_{2}(t) v_{x y}(t, x)=r v(t, x)$, 
on $(t, x, y) \in[0, T) \times \mathbb{R}^{2}$, with

$$
\begin{aligned}
& \Psi_{1}(t)=\sigma(t) e^{-\int_{0}^{t} \alpha(u) d u} \int_{0}^{t} \phi(t, s) \sigma(s) e^{-\int_{0}^{s} \alpha(u) d u} d s \\
& \Psi_{2}(t)=\sigma(t) \int_{0}^{t} \phi(t, s) \sigma(s) \int_{s}^{t} e^{-\int_{s}^{u} \alpha(\tau) d \tau} d u d s,
\end{aligned}
$$

and terminal condition $v(T, x, y)=f(y)$. If $f\left(\int_{0}^{T} X(s) d s\right), v\left(t, X(t), \int_{0}^{t} X(s) d s\right) \in L^{2}(Q)$ for $t \in[0, T]$, where $Q$ is the equivalent martingale measure leading to (3.5), then

$$
v\left(t, X(t), \int_{0}^{t} X(s) d s\right)=e^{-r(T-t)} \widehat{E}_{Q}\left[f\left(\int_{0}^{T} X(s) d s\right) \mid \mathcal{F}_{t}^{H}\right] .
$$

Proof. Assume without loss of generality that $r=0$. To make the calculations more transparent, we introduce the following functions, $k(t)=\mathrm{e}^{\int_{0}^{t} \alpha(s) d s}$ and $K(t)=\int_{0}^{t} k^{-1}(s) d s$. From (3.6) under $Q$ we easily find

$$
\int_{0}^{t} X(s) d s=\int_{0}^{t} a(s) k^{-1}(s) d s+K(t) \int_{0}^{t} \sigma(s) k(s) \widetilde{B}_{H}(s)-\int_{0}^{t} \sigma(s) K(s) k(s) \widetilde{B}_{H}(s),
$$

where

$$
a(t)=x+\int_{0}^{t} \alpha(s)(\lambda(s)+\theta(s)) \mathrm{e}^{\int_{0}^{s} \alpha(u) d u} d s .
$$

Remark that $a(t)$ is the same as in the proof of Proposition 6.1. Let us further introduce the notation

$$
Z_{1}(t)=\int_{0}^{t} \sigma(s) k(s) \widetilde{B}_{H}(s), \quad Z_{2}(t)=\int_{0}^{t} \sigma(s) K(s) k(s) \widetilde{B}_{H}(s) .
$$

We can now write (using calculations made in the proof of Proposition 6.1)

$$
v\left(t, X(t), \int_{0}^{t} X(s) d s\right)=v\left(t, k^{-1}\left(a(t)+Z_{1}(t)\right), \int_{0}^{t} a(s) k^{-1}(s) d s+K(t) Z_{1}(t)-Z_{2}(t)\right),
$$

or

$$
v(t, x, y)=v\left(t, k^{-1}\left(a(t)+z_{1}\right), \int_{0}^{t} a(s) k^{-1}(s) d s+K(t) z_{1}-z_{2}\right) .
$$

We are going to use the multidimensional Itô Formula in Corollary 2.4. In order to use this, we need the derivatives of $v$ with respect to $t, z_{1}$ and $z_{2}$ : We list them here for convenience:

$$
\begin{aligned}
v_{z_{1}} & =k^{-1}(t) v_{x}+K(t) v_{y}, \quad v_{z_{2}}=-v_{y} \\
v_{z_{1} z_{1}} & =k^{-2}(t) v_{x x}+2 K(t) k^{-1}(t) v_{x y}+K^{2}(t) v_{y y} \\
v_{z_{1} z_{2}} & =v_{z_{2} z_{1}}=-k^{-1}(t) v_{x y}-K(t) v_{y y} \\
v_{z_{2} z_{2}} & =v_{y y} .
\end{aligned}
$$

The Itô Formula in Corollary 2.4 now yields,

$$
v\left(t, X(t), \int_{0}^{t} X(s) d s\right)=v(0, x, 0)
$$




$$
\begin{aligned}
& +\int_{0}^{t} v_{t}+v_{x}\left(-\alpha(s) k^{-1}(s)\left(a(s)+Z_{1}(s)\right)+k^{-1}(s) \alpha(s)(\lambda(s)+\theta(s)) k(s)\right) d s \\
& +\int_{0}^{t} v_{y}\left(a(t) k^{-1}(t)+k^{-1} Z_{1}(t)\right) d s+\int_{0}^{t}\left(k^{-1}(t) v_{x}+K(t) v_{y}\right) \sigma(s) k(s) d \widetilde{B}_{H}(s) \\
& -\int_{0}^{t} v_{y} \sigma(s) K(s) k(s) d \widetilde{B}_{H}(s) \\
& +\int_{0}^{t}\left(k^{-2}(s) v_{x x}+2 k^{-1}(s) K(s) v_{x y}+K^{2}(s) v_{y y}\right) \sigma(s) k(s) \int_{0}^{s} \phi(s, \tau) \sigma(\tau) k(\tau) d \tau d s \\
& -\int_{0}^{t}\left(k^{-1}(s) v_{x y}+K(s) v_{y y}\right) \sigma(s) k(s) \int_{0}^{t} \phi(s, \tau) \sigma(\tau) k(\tau) K(\tau) d \tau d s \\
& -\int_{0}^{t}\left(k^{-1}(s) v_{x y}+K(s) v_{y y}\right) \sigma(s) k(s) K(s) \int_{0}^{t} \phi(s, \tau) \sigma(\tau) k(\tau) d \tau d s \\
& +\int_{0}^{t} v_{y y} \sigma(s) k(s) K(s) \int_{0}^{s} \phi(s, \tau) \sigma(\tau) k(\tau) K(\tau) d \tau d s \\
& =v(0, x, 0)+\int_{0}^{t} v_{t}+\alpha(s)(\lambda(s)+\theta(s)-X(s)) v_{x}+X(s) v_{y} d s \\
& +\int_{0}^{t} \sigma(s)\left(v_{x}+2 k(s) K(s) v_{y}\right) d \widetilde{B}_{H}(s)+\int_{0}^{t} v_{x x} \Psi \Psi_{1}(s)+v_{x y} \Psi_{2}(s) d s \\
& =v(0, x, 0)+\int_{0}^{t} \sigma(s)\left(v_{x}+2 k(s) K(s) v_{y}\right) d \widetilde{B}_{H}(s),
\end{aligned}
$$

since $v$ solves the partial differential equation (6.2). By assumption on $v_{x}, v_{y}, \sigma$ and $\alpha$, the fractional Itô integral on the right-hand side is a quasi-martingale (since it is square integrable). By using the result of Itô's Formula for $t=T$, and subtracting for $t$, we find

$$
v\left(T, X(T), \int_{0}^{T} X(s) d s\right)-v\left(t, X(t), \int_{0}^{t} X(s) d s\right)=\int_{t}^{T} \sigma(s)\left(v_{x}+2 k(s) K(s) v_{y}\right) d \widetilde{B}_{H}(s)
$$

By the terminal condition, $v\left(T, X(T), \int_{0}^{T} X(s) d s\right)=f\left(\int_{0}^{T} X(s) d s\right)$. Using the adaptedness of $v\left(t, X(t), \int_{0}^{t} X(s) d s\right)$, we find after taking the quasi-conditional expectation on both sides

$$
\widehat{\mathrm{E}}_{Q}\left[f\left(\int_{0}^{T} X(s) d s\right) \mid \mathcal{F}_{t}^{H}\right]-v\left(t, X(t), \int_{0}^{t} X(s) d s\right)=0 .
$$

This proves the Proposition.

Note the term $\Psi_{2}(t) v_{x y}$ in (6.2). When $H=0.5$, this term cancels out, but for $H>0.5$ $\Psi_{2}$ is in general different from zero.

The results in the this Section and the previous one partly confirm the conjecture of Brody, Syroka and Zervos [3] on the dynamics of the price of a weather claim. They claimed it should solve a Black \& Scholes type of partial differential equation, however, 
they based their conjecture on the classical conditional expectation. We use the quasiconditional expectation to derive arbitrage-free pricing dynamics which can be calculated via a Fourier transform or as the solution of a modulated Black \& Scholes partial differential equation. Our results coincides of course with those of Brody, Syroka and Zervos [3] when we consider the price at time 0 , that is, when the contract is entered. This is the only instant when the conditional and quasi-conditional expectations coincide.

\section{REFERENCES}

[1] Benth, F. E. and Potthoff, J. (1996), On the martingale property for generalized stochastic processes, Stoch. Stoch. Reports Vol 58, 349-367.

[2] Biagini, F. and Øksendal, B. (2002), Minimal variance hedging for fractional Brownian motion, Preprint No. 1, University of Oslo, Norway.

[3] Brody, D. C., Syroka, J. and Zervos, M. (2002), Dynamical pricing of weather derivatives, Quant. Finance, Vol 3, 189-198.

[4] Davis, M. H. A. (2001), Pricing weather derivatives by marginal value. Quant. Finance 1, 305-308.

[5] Folland, G. B. (1984), Real Analysis, John Wiley \& Sons.

[6] Hida, T. (1980), Brownian motion, Springer Verlag.

[7] Hida, T, Kuo, H.-H., Potthoff, J. and Streit, L. (1993): White Noise - An infinte dimensional calculus, Kluwer Academic Publishers.

[8] Duncan, T. E., Hu, Y. and Pasik-Duncan, B. (2000), Stochastic calculus for fractional Brownian motion I. Theory., SIAM J. Control Optim. 38, pp. 582-612.

[9] Hu, Y. and Øksendal, B. (1999), Fractional white noise calculus and applications to finance. Preprint No. 10, University of Oslo, Norway.

[10] Hu, Y. and Øksendal, B. (2002), Chaos expansion of local time of fractional Brownian motions, Stoch Anal. Appl. 20(4), 815-837.

[11] Hu, Y., Øksendal, B. and Salopek, D. M. (2001), Weighted local times for fractional Brownian motion and applications to finance. Preprint No. 13, University of Oslo, Norway.

[12] Kopp, L. (2003), Master thesis, University of Oslo, Norway.

[13] Lundgren, T. and Chiang, D. (1967), Solution of a class of singular integral equations, Quart. J. Appl. Math. 24, 303-313.

[14] Necula, C. (2002), Option pricing in a fractional Brownian motion environment. Preprint, Academy of Econ. Studies, Bucharest, Rumania.

(Fred Espen Benth)

Department of Mathematics

UNIVERSITY OF OsLo

P.O. Box 1053, Blindern

N-0316 Oslo, Norway

E-mail address: fredb@math.uio.no

URL: http: //www .math . uio.no/ "fredb/ 